\title{
Fetal Heart Acceleration
}

National Cancer Institute

\section{Source}

National Cancer Institute. Fetal Heart Acceleration. NCI Thesaurus. Code C92788.

An abrupt increase is defined as an increase from the onset of acceleration to the peak in less than 30 seconds. To be called an acceleration, the peak must be greater than or equal to $15 \mathrm{bpm}$, and the acceleration must last greater than or equal to 15 seconds from the onset to return to baseline. 